\title{
Stringy instability inside the black hole
}

\section{Nissan Itzhaki}

Physics Department, Tel-Aviv University, Ramat-Aviv, 69978, Israel

E-mail: nitzhaki@post.tau.ac.il

ABSTRACT: We show that negative $(\nabla \Phi)^{2}$, where $\Phi$ is the dilaton, leads to a rapid creation of folded strings. Consequently it appears that the interior of the $\operatorname{SL}(2, \mathbb{R})_{k} / \mathrm{U}(1)$ black hole is not empty, but is filled with folded strings.

Keywords: 2D Gravity, Black Holes, Black Holes in String Theory, Long strings

ArXiv EPrint: 1808.02259 
Motivated by [1] we speculated sometime ago [2] that a concrete way to express the challenge in having a non trivial structure at the horizon of a large Black Hole $(\mathrm{BH})$ is to be able to write down an effective action that renders the horizon special. We claimed that such an effective action must involve a "horizon order parameter"; an operator whose expectation value indicates if we are inside or outside the $\mathrm{BH}$. The horizon order parameter meant to be a trigger that modifies the physics inside the $\mathrm{BH}$ considerably compared to the standard physics outside the BH.

Recently [3] it was argued that in the case of the $2 \mathrm{D} \mathrm{SL}(2, \mathbb{R})_{k} / \mathrm{U}(1) \mathrm{BH}[4-6]$ the horizon order parameter might take a particularly simple form

$$
\mathcal{O}=(\nabla \Phi)^{2}
$$

where $\Phi$ is the dilaton. Outside the $\operatorname{SL}(2, \mathbb{R})_{k} / \mathrm{U}(1) \mathrm{BH}$ the operator $\mathcal{O}$ is positive while inside the $\mathrm{BH}$ it is negative.

Some indirect evidence from the exact reflection coefficient of [7] was provided that the $\mathrm{SL}(2, \mathbb{R})_{k} / \mathrm{U}(1) \mathrm{BH}$ interior is not empty in classical string theory $[3,8]$. These papers, however, did not explain how come the $\mathrm{SL}(2, \mathbb{R})_{k} / \mathrm{U}(1) \mathrm{BH}$ is not empty or what it is filled with. Moreover no relation with $\mathcal{O}$ was established. In particular it was not clear how the fact that $\mathcal{O}$ flips sign when crossing the horizon could possibly trigger non trivial effects inside the $\mathrm{BH}$.

In this short note we attempt to fill up this gap. We argue that when $\mathcal{O}$ is negative folded strings are created rapidly. As a result the $\mathrm{SL}(2, \mathbb{R})_{k} / \mathrm{U}(1) \mathrm{BH}$ interior is not empty but is filled with folded strings. The idea that the $\mathrm{BH}$ is made out of fundamental strings is not new $[9,10]$. However, in the past these claims where made about small, stringy size, BHs while here we discuss large BHs.

The classical background associated with the $\mathrm{SL}(2, \mathbb{R})_{k} / \mathrm{U}(1) \mathrm{BH}$ is (we work with $\left.\alpha^{\prime}=1\right)$

$$
d s^{2}=-f(r) d t^{2}+\frac{d r^{2}}{f(r)}, \quad \Phi(r)=\phi_{0}-Q r
$$

where $f(r)=1-\mu e^{-2 Q r}$. We focus on the supersymmetric case that describes the near horizon limit of $k$ NS5-branes in type II strings [26]. We consider the limit where, $Q=$ $1 / \sqrt{k}$, is small. In this limit the curvature is small away from the singularity. We also take $\phi_{0} \rightarrow-\infty$ so that stringy loops can be neglected. The effect we describe happens already in classical string theory.

Away from the BH, when $r \rightarrow \infty$, we have a 2D linear dilaton background

$$
d s^{2}=-d t^{2}+d r^{2}, \quad \Phi=-Q r
$$

with $Q>0$. As far as the small fluctuations goes the length scale associated with the linear dilaton is $1 / Q$. This is evident from the background eq. (3) and also from the fact that the conformal dimension of an operator with momentum $p$ is shifted from $p^{2} / 4$ to $p(p+Q) / 4$.

There are also classical long string solutions in this background [11]. An interesting aspect of these solutions, that will play a key role below, is that the relevant length scale 


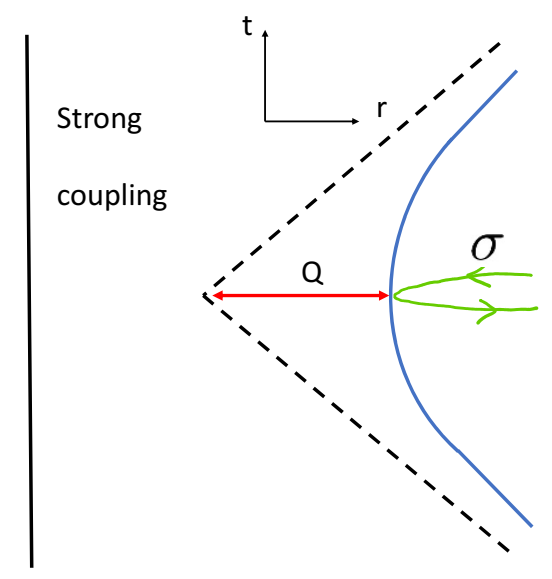

Figure 1. The shape of the long folded string of [11]. The green line indicates the way the string folds which is always towards the weak coupling region. At early and late times the tip of the string is moving at a speed that approaches \pm 1 .

associated with them is not $1 / Q$, but $Q$. Let us review their construction. Consider a classical string that propagates non trivially in the background eq. (3). Working in the temporal gauge $t=\tau$ the equation of motions are $\partial_{+} \partial_{-} r=0$ and the Virasoro constraints read

$$
-1+\left(\partial_{+} r\right)^{2}+Q \partial_{+}^{2} r=0, \quad-1+\left(\partial_{-} r\right)^{2}+Q \partial_{-}^{2} r=0 .
$$

The solution to these equations is

$$
r=r_{0}+Q \log \left(\frac{1}{2}\left(\cosh \left(\frac{t-t_{0}}{Q}\right)+\cosh \left(\frac{\sigma}{Q}\right)\right)\right) .
$$

This solution is not periodic. It describes an infinity long folded string that is stretched all the way to the weak coupling region.

The turning point of the string, where it is at rest, is at $r=r_{0}$ and $t=t_{0}$. At $t$ much smaller or larger than $t_{0}$ the tip of the string is moving at a speed that is approaching the speed of light (see figure 1). Note that unlike the yo-yo solution of [12-14] this solution is a smooth solution that does not involve discontinuities in $\partial_{ \pm} r$.

The total energy of such a string is infinite. The reason for the absent of finite energy classical string configurations is that no matter how small $Q$ is the linear dilaton prevents the string from folding towards the strong coupling. To see how this comes about it is instructive to consider eq. (4) at the turning point where the velocity vanishes and the Virasoro constraint are approximated by

$$
\partial_{+}^{2} r=\frac{1}{Q}, \quad \partial_{-}^{2} r=\frac{1}{Q} .
$$

This implies that near the turning point there is a constant acceleration, that scales like $1 / Q$. One way to think about this acceleration is that the slope of the dilaton induces a mass of the order of $Q$ at the tip of the string folds [11]. Since we work in units in which the string tension is 1 Newton's second law implies eq. (6) which leads to

$$
r=\frac{1}{2 Q}\left(\tau^{2}+\sigma^{2}\right)
$$

This shows that the string can fold only in one direction, towards the weak coupling region. 
The exact solution eq. (5) as well as the approximated solution eq. (6) imply that the length scale associated with the long string is $Q$ which for $Q \ll 1$ is much smaller than the perturbative scale, $1 / Q$, associated with eq. (3). The fact that the non-perturbative long string introduces a new scale to the problem is closely related to the fact that the reflection coefficient in Liouville theory [15] and in the $\mathrm{SL}(2, \mathbb{R})_{k}$ model [7] involve the non-perturbative momentum scale, $1 / Q$ ontop of the perturbative scale $Q$.

What happens when we approach the $\mathrm{BH}$ ? Is it possible that outside the $\mathrm{BH}$, where the curvature is of the order of $(\nabla \Phi)^{2}$ and eq. (3) is not a good approximation, there are finite energy classical configurations? There is a simple argument why, at least for small $Q$, the answer is no. To have a finite energy configuration the string should be able to turn both towards the $\mathrm{BH}$ and away from the $\mathrm{BH}$. As discussed above the length scale associated with the turning of the string is $Q$. Curvature effects, that are absent in the discussion above, are important at much larger scales that are of the order of $1 / Q$. Therefore, at least for small $Q$, the curvature associated with the solution eq. (2) cannot change the conclusion that outside the $\mathrm{BH}$ the string can turn only towards the weak coupling region and hence there are only infinitely long string configurations outside the BH.

Next we wish to explore what happens inside the $\operatorname{SL}(2, \mathbb{R})_{k}$ BH where $(\nabla \Phi)^{2}<0$. First we consider a useful toy model, that also have $(\nabla \Phi)^{2}<0$ - a time-like linear dilaton. The background takes the form

$$
d s^{2}=-\left(d X^{0}\right)^{2}+\left(d X^{1}\right)^{2}, \quad \Phi=Q X^{0} .
$$

Together with a matter CFT with a central charge such that the total central charge is 15 this is an exact background in classical superstring theory. The matter theory plays no role in our discussion. We take $Q>0$ so that, just like in the dynamically formed $\mathrm{BH}$, the strong coupling is in the future. ${ }^{1}$

The gauge $X^{0}=\tau$ dismisses the effect of the linear dilaton. In this gauge the only solution, that does not involve discontinuous $\partial_{ \pm} X^{1}$, is the trivial one $X^{1}=\sigma$. There are, however, non-trivial smooth solutions that are sensitive to the time-like linear dilaton. To reveal them we work in the unusual gauge $X^{1}=\sigma$. Then the Virasoro constraints are

$$
1-\left(\partial_{+} X^{0}\right)^{2}-Q \partial_{+}^{2} X^{0}=0, \quad 1-\left(\partial_{-} X^{0}\right)^{2}-Q \partial_{-}^{2} X^{0}=0
$$

and the solution is

$$
X^{0}=x^{0}+Q \log \left(\frac{1}{2}\left(\cosh \left(\frac{X^{1}-x^{1}}{Q}\right)+\cosh \left(\frac{\tau}{Q}\right)\right)\right) .
$$

Despite the technical similarity with eq. (5) the physics associated with this solution is quite different than that of eq. (5). The string is created from the vacuum at a certain time $x^{0}$ and place $x^{1}$ and is folded towards the strong coupling (see figure 2). At the tip the string is moving at an infinite speed that, for small $Q$, is quickly reduced towards the speed of light.

\footnotetext{
${ }^{1}$ When considering time-like linear dilaton as a toy model for cosmology, e.g. [16, 17], it is natural to have the strong coupling in the past.
} 


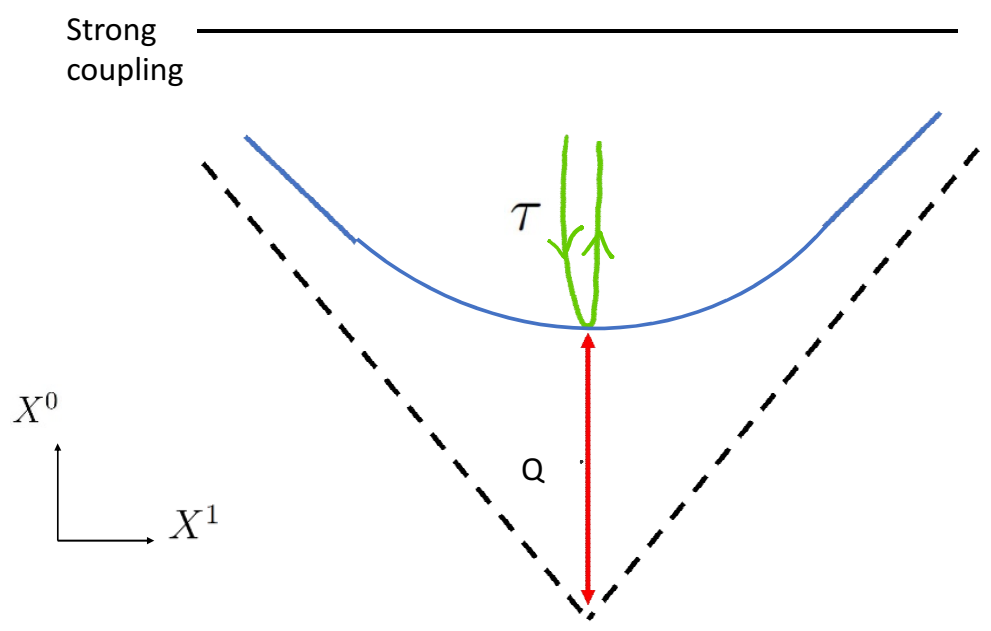

Figure 2. The green line indicates the way the string folds which is always towards the strong coupling region. The tip of the string is always moving faster than light. It approaches the speed of light, the dashed lines, when $\left|X_{1}\right|$ is large.

At first sight, figure 2, that describes eq. (10), appears to resemble figure 3 that describes the Schwinger mechanism [18] or more precisely its stringy generalization (see e.g. [19]). There are, however, crucial differences. First, eq. (10) is a classical solution in Minkowski signature. A semi-classical description of the Schwinger mechanism that starts from the vacuum and ends with on-shell particles involves gluing a Minkowskian solution with a Euclidean solution (see figure 3). The Euclidean section implies that, unlike in eq. (10), the Schwinger mechanism is a quantum process that involves tunneling and is exponentially suppressed by $S_{E} / \hbar$. The lack of a Euclidean section in eq. (10) implies that the creation rate associated with it is not expected to be suppressed exponentially.

Second, in the Schwinger mechanism the particles (or strings) are moving slower than light. This is not the case in eq. (10). The points where the string folds are moving faster than light. This suggests the following as the mechanism behind this solution. In analogy with [11] the linear dilaton induces a mass at the point where the string folds. Only that now since we have a time-like linear dilaton the mass is tachyonic, $m_{t i p}^{2} \sim-Q^{2}$. Condensation of this tachyon attempts to generate a runaway behaviour. It is the tension of the string that holds the configuration together. The balance between the two is what drives eq. (10) and induces the large momentum scale, $1 / Q$, associated with it.

We do not know what is the end-point of the condensation of this classical configuration. It would be particularly interesting if it leads to a resolution of the future strong coupling singularity.

We are now in a position to describe the folded string inside the $\mathrm{SL}(2, \mathbb{R})_{k} / \mathrm{U}(1) \mathrm{BH}$. The slope of the time-like dilaton inside the $\mathrm{SL}(2, \mathbb{R})_{k} / \mathrm{U}(1) \mathrm{BH}$ is not constant and the metric is not flat. Hence eq. (8) does not describe the region behind the horizon. However, $(\nabla \Phi)^{2}$ is negative there and we saw that the length scale associated with the folded string creation is $Q$. This implies that inside the $\mathrm{SL}(2, \mathbb{R})_{k} / \mathrm{U}(1) \mathrm{BH}$ the length scale associated with the creation of the folded string is $\sqrt{-(\nabla \Phi)^{2}}$. This scale is typically of order $1 / \sqrt{k}$ 


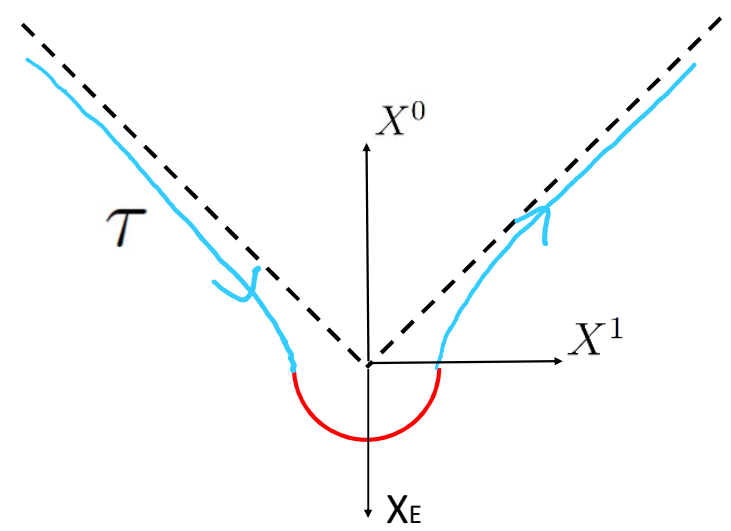

Figure 3. The Schwinger mechanism. The red line represents the Euclidean solution. It is glued at $X_{E}=X^{0}=0$ to the Minkowskian solution that is represented by the blue lines.

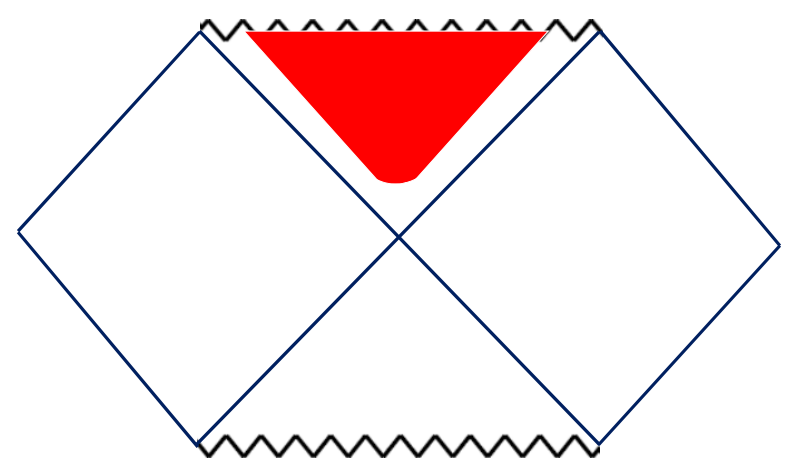

Figure 4. A typical folded string inside the $\mathrm{SL}(2, \mathbb{R})_{k} / \mathrm{U}(1) \mathrm{BH}$.

which is much smaller than the curvature scale $\sqrt{k}$. Hence in the large $k$ limit the folded string creation is, for all practical purposes, a local process. This means that the BH curvature cannot modify much the creation of the folded string. It will surely modify eq. (10) at distances of order $\sqrt{k}$, but not the conclusion that such folded strings are created. We expect a typical folded string inside the $\mathrm{SL}(2, \mathbb{R})_{k} / \mathrm{U}(1) \mathrm{BH}$ to take the shaped presented in figure 4.

The folded string condensation will not stop until $(\nabla \Phi)^{2}$ is not negative everywhere inside the $\mathrm{BH}$. This means that the entire $\mathrm{BH}$ interior should be filled with folded strings. This appears to be the case both for eternal and dynamically formed $\operatorname{SL}(2, \mathbb{R})_{k} / \mathrm{U}(1) \mathrm{BH}$.

This conclusion seems to fit neatly with claims made in $[3,8]$ that the potential associated with the $\mathrm{SL}(2, \mathbb{R})_{k} / \mathrm{U}(1) \mathrm{BH}$ blows up just behind the horizon. The unusual properties of eq. (10) suggests that its backreaction is likely to be non-standard and could drastically modify the $\mathrm{BH}$ interior. It remains to be seen if precise connection with the potential found in $[3,8]$ can be made. The fact that the potential behind the horizon found in $[3,8]$ is determined by the same length scale, $1 / \sqrt{k}$, that controls eq. (10) seems to support this.

The conclusion that the interior of the eternal $\mathrm{SL}(2, \mathbb{R})_{k} / \mathrm{U}(1) \mathrm{BH}$ is filled with strings also seems to go well with some Euclidean reasoning. The FZZ duality [20,21] implies that at the tip of the cigar there is a condensation of a winding one tachyon mode $[22,23]$. The Harlte Hawking wave function [24] then seems to imply that the BH interior should be filled 


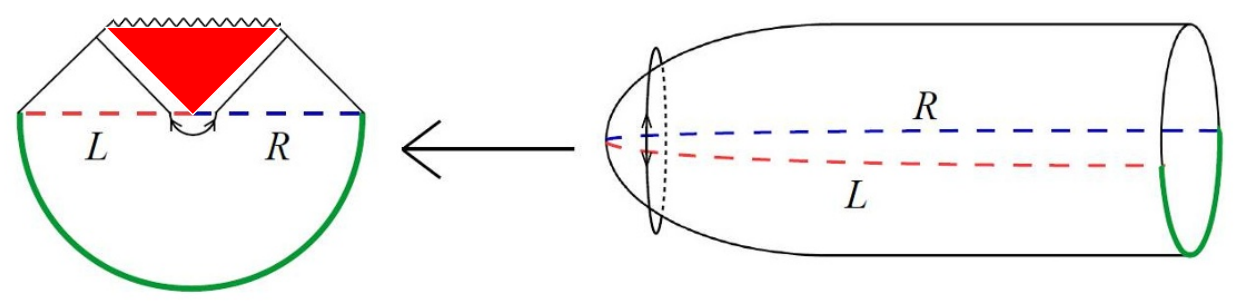

Figure 5. The winding one tachyon mode that condenses at the tip of the cigar appears to be related to the folded string that fills up the entire $\mathrm{SL}(2, \mathbb{R})_{k} / \mathrm{U}(1) \mathrm{BH}$ interior.

with strings (see figure 5). It seems reasonable to suspect that the wave function of the winding one tachyon mode found in [25] is related to the wave function of the folding point of the string discussed here. One needs to go beyond the classical discussion presented here to study this.

The $\mathrm{SL}(2, \mathbb{R})_{k} / \mathrm{U}(1) \mathrm{BH}$ is the near horizon of $k$ NS5-branes [26]. The fundamental string is the electric-magnetic dual of the NS5-brane [27]. It is, therefore, natural to speculate that the region behind the horizon of near extremal Dp-branes is filled with their electric-magnetic dual, D(6-p)-branes [28].

In this note we focused on BHs. It is likely, however, that the folded string creation could have interesting applications to cosmology as well.

\section{Acknowledgments}

We thank K. Attali, A. Giveon and L. Liram for discussions. This work is supported in part by the I-CORE Program of the Planning and Budgeting Committee and the Israel Science Foundation (Center No. 1937/12), and by a center of excellence supported by the Israel Science Foundation (grant number 1989/14).

Open Access. This article is distributed under the terms of the Creative Commons Attribution License (CC-BY 4.0), which permits any use, distribution and reproduction in any medium, provided the original author(s) and source are credited.

\section{References}

[1] N. Itzhaki, Is the black hole complementarity principle really necessary?, hep-th/9607028 [INSPIRE].

[2] N. Itzhaki, The horizon order parameter, hep-th/0403153 [INSPIRE].

[3] N. Itzhaki and L. Liram, A stringy glimpse into the black hole horizon, JHEP 04 (2018) 018 [arXiv: 1801.04939] [INSPIRE].

[4] S. Elitzur, A. Forge and E. Rabinovici, Some global aspects of string compactifications, Nucl. Phys. B 359 (1991) 581 [inSPIRE].

[5] G. Mandal, A.M. Sengupta and S.R. Wadia, Classical solutions of two-dimensional string theory, Mod. Phys. Lett. A 6 (1991) 1685 [inSPIRE]. 
[6] E. Witten, On string theory and black holes, Phys. Rev. D 44 (1991) 314 [inSPIRE].

[7] J. Teschner, Operator product expansion and factorization in the $H+(3)$ WZNW model, Nucl. Phys. B 571 (2000) 555 [hep-th/9906215] [inSPIRE].

[8] R. Ben-Israel, A. Giveon, N. Itzhaki and L. Liram, On the black hole interior in string theory, JHEP 05 (2017) 094 [arXiv: 1702.03583] [INSPIRE].

[9] L. Susskind, Some speculations about black hole entropy in string theory, hep-th/9309145 [INSPIRE].

[10] G.T. Horowitz and J. Polchinski, A correspondence principle for black holes and strings, Phys. Rev. D 55 (1997) 6189 [hep-th/9612146] [INSPIRE].

[11] J.M. Maldacena, Long strings in two dimensional string theory and non-singlets in the matrix model, JHEP 09 (2005) 078 [hep-th/0503112] [INSPIRE].

[12] W.A. Bardeen, I. Bars, A.J. Hanson and R.D. Peccei, A study of the longitudinal kink modes of the string, Phys. Rev. D 13 (1976) 2364 [inSPIRE].

[13] W.A. Bardeen, I. Bars, A.J. Hanson and R.D. Peccei, Quantum Poincaré covariance of the D = 2 string, Phys. Rev. D 14 (1976) 2193 [InSPIRE].

[14] I. Bars, Folded strings, hep-th/9412044 [INSPIRE].

[15] A.B. Zamolodchikov and A.B. Zamolodchikov, Structure constants and conformal bootstrap in Liouville field theory, Nucl. Phys. B 477 (1996) 577 [hep-th/9506136] [INSPIRE].

[16] S. Hellerman and I. Swanson, Cosmological solutions of supercritical string theory, Phys. Rev. D 77 (2008) 126011 [hep-th/0611317] [INSPIRE].

[17] O. Aharony and E. Silverstein, Supercritical stability, transitions and (pseudo)tachyons, Phys. Rev. D 75 (2007) 046003 [hep-th/0612031] [InSPIRE].

[18] J.S. Schwinger, On gauge invariance and vacuum polarization, Phys. Rev. 82 (1951) 664 [INSPIRE].

[19] F. Dowker, J.P. Gauntlett, G.W. Gibbons and G.T. Horowitz, Nucleation of p-branes and fundamental strings, Phys. Rev. D 53 (1996) 7115 [hep-th/9512154] [INSPIRE].

[20] V.A. Fateev, A.B. Zamolodchikov and Al.B. Zamolodchikov, unpublished.

[21] V. Kazakov, I.K. Kostov and D. Kutasov, A matrix model for the two-dimensional black hole, Nucl. Phys. B 622 (2002) 141 [hep-th/0101011] [InSPIRE].

[22] D. Kutasov and D.A. Sahakyan, Comments on the thermodynamics of little string theory, JHEP 02 (2001) 021 [hep-th/0012258] [INSPIRE].

[23] J. Maldacena, Comments on the two dimensional black hole, talk given at Strings 2004, June 28-July 2, Paris, France (2004).

[24] J.B. Hartle and S.W. Hawking, Path integral derivation of black hole radiance, Phys. Rev. D 13 (1976) 2188 [INSPIRE].

[25] A. Giveon and N. Itzhaki, String theory at the tip of the cigar, JHEP 09 (2013) 079 [arXiv: 1305.4799] [INSPIRE].

[26] J.M. Maldacena and A. Strominger, Semiclassical decay of near extremal five-branes, JHEP 12 (1997) 008 [hep-th/9710014] [INSPIRE].

[27] C.G. Callan Jr., J.A. Harvey and A. Strominger, Supersymmetric string solitons, hep-th/9112030 [INSPIRE].

[28] J. Polchinski, Dirichlet branes and Ramond-Ramond charges, Phys. Rev. Lett. 75 (1995) 4724 [hep-th/9510017] [INSPIRE]. 